\title{
Design of Robust Observer-Based Backstepping Control for a Satellite Control System
}

\author{
Saleh Alshamali (D) and Elham Aljuwaiser \\ Department of Electrical Engineering, Kuwait University, P.O. Box 5969, Safat 13060, Kuwait \\ Correspondence should be addressed to Saleh Alshamali; s.alshamali@ku.edu.kw
}

Received 12 February 2019; Revised 23 April 2019; Accepted 9 May 2019; Published 26 May 2019

Academic Editor: Xue-Jun Xie

Copyright (c) 2019 Saleh Alshamali and Elham Aljuwaiser. This is an open access article distributed under the Creative Commons Attribution License, which permits unrestricted use, distribution, and reproduction in any medium, provided the original work is properly cited.

\begin{abstract}
This paper presents the attitude tracking of a class of satellite control systems under external disturbances. Once the error dynamics of the satellite are obtained, a nonlinear transformation expresses them in a suitable representation for the design of a high-gain observer and backstepping control. The observer-based backstepping controller is then designed to drive the angles of the satellite dynamics to their desired values in the presence of exogenous disturbances. Closed-loop stability of the proposed controller is demonstrated via Lyapunov theory, and its effectiveness is confirmed through numerical simulations.
\end{abstract}

\section{Introduction}

A great number of satellite systems are orbiting Earth for various purposes. These satellites are often classified in terms of their orbit, such as low Earth orbit, medium Earth orbit, geostationary orbits, and geosynchronous orbits [1, 2]. Alternatively, they can be classified according to their mechanism of control torque generation, such as thrusters, reaction wheels, magnetic torquer rods, and control moment gyroscopes [3-5]. The design of attitude tracking control for satellite systems poses challenges to engineers, especially when the satellite is under the influence of external disturbances. Therefore, a successful control design must ensure stability of the closed-loop system.

Several strategies have been proposed for satellite control systems (SCSs). For instance, actuator failure compensation via adaptive backstepping control has been addressed in [6]. In addition, a nonsingular terminal sliding mode control has been proposed for attitude tracking of small satellites by using a combined energy and attitude control system [7]. Likewise, magnetic attitude control has been developed for SCSs with uneven inertial distributions by combining Kalman filtering and feedforward control to estimate and reject external disturbances [8]. Nonlinear sliding mode control for attitude tracking of a magnetically actuated satellite is introduced in [9]. More recently, a three-stage sliding mode control for attitude tracking of a satellite system with uncertain inertia matrix and torque disturbance has been proposed [10]. Attitude control of a rigid body with uncertain inertia matrix using sliding mode control along with a state observer has also been addressed [11]. In [12], nonlinear model predictive control is applied to a flexible satellite, where the controller parameters are optimized by a genetic algorithm. Satellite attitude stabilization has also been addressed using the statedependent Riccati equation control method [13]. Horri et al. [14] developed a gain-scheduled controller for a SCS based on inverse optimal control to improve the settling time of a benchmark proportional-derivative controller. Attitude control has also been addressed using an adaptive controller based on a radial basis function neural network [15]. A faulttolerant control law based on sliding mode control has been proposed for a SCS under solar perturbations and actuator faults [16]. Xiao et al. [17] addressed attitude tracking of a flexible spacecraft via an adaptive sliding mode backstepping controller.

Unlike previous studies, this work combines backstepping control with a high-gain observer for attitude tracking of a SCS under external disturbances. Backstepping control is a simple, flexible, and versatile approach that provides 
high stability and robustness. Moreover, the use of a highgain observer offers advantages over many other nonlinear observers. Notably, such observer has a simple design, fast convergence, and guaranteed stability with the appropriate choice of eigenvalues. Nevertheless, to fully exploit this observer, the satellite dynamics must be expressed in feedback form. Therefore, a nonlinear transformation is proposed in this paper to achieve this representation, which is suitable for appropriate observer design.

The contributions in this paper are threefold. First, a system transformation is proposed for the dynamics of the SCS to obtain a feedback form. Second, a high-gain observer is designed to estimate the rotational angles and angular velocities of the SCS. Third, a robust backstepping control law is derived for the satellite angles to track a reference trajectory under external disturbances. The remainder of this paper is organized as follows. The kinematic and dynamic models of the SCS considered in this study are presented in Section 2. In Section 3, the error dynamics are formulated, and a nonlinear transformation is introduced to enable the use of the highgain observer and to facilitate the design of the backstepping controller. Section 4 describes high-gain observers, which are the basis for the robust backstepping controller introduced in Section 5. Simulation results of the proposed controller are reported in Section 6. Finally, some concluding remarks are given in Section 7.

\section{Kinematic and Dynamic Models}

The kinematic model of the SCS is given by [6]

$$
\begin{aligned}
& \dot{\theta}_{\mathrm{p}}=\omega_{y} \sin \left(\theta_{\mathrm{r}}\right)+\omega_{z} \cos \left(\theta_{\mathrm{r}}\right), \\
& \dot{\theta}_{\mathrm{y}}=\frac{\left(\omega_{y} \cos \left(\theta_{\mathrm{r}}\right)-\omega_{z} \sin \left(\theta_{\mathrm{r}}\right)\right)}{\cos \left(\theta_{\mathrm{p}}\right)}, \\
& \dot{\theta}_{\mathrm{r}}=\omega_{x}-\tan \left(\theta_{\mathrm{p}}\right)\left(\omega_{y} \cos \left(\theta_{\mathrm{r}}\right)-\omega_{z} \sin \left(\theta_{\mathrm{r}}\right)\right),
\end{aligned}
$$

where $\theta_{\mathrm{p}}, \theta_{\mathrm{y}}$, and $\theta_{\mathrm{r}}$ represent the pitch, yaw, and roll angles of the satellite, respectively, and $\omega_{x}, \omega_{y}$, and $\omega_{z}$ are the satellite angular velocities around the corresponding axis with respect to the inertial frame.

Assumption 1. The rotational angles vary in a small range, and the satellite does not perform vertical maneuvers.

Define the state vectors as $\theta=\left[\theta_{\mathrm{p}} \theta_{\mathrm{y}} \theta_{\mathrm{r}}\right]^{\mathrm{T}}$ and $\omega=$ $\left[\omega_{x} \omega_{y} \omega_{z}\right]^{\mathrm{T}}$. The uncertain dynamic model of the SCS is given by

$$
J \dot{\omega}=-\omega^{*} J \omega+D u(t)+F_{\mathrm{d}}(\omega(t), t),
$$

where $J \in \mathfrak{R}^{3 \times 3}$ is a symmetric positive definite inertia matrix, $D \in \mathfrak{R}^{3 \times 3}$ is the actuator distribution matrix assumed to have an orthogonal configuration (i.e., $D=I_{3 \times 3}$ ),
$F_{\mathrm{d}}(\omega(t), t)$ is the vector of external disturbances, and $\omega^{*}$ is a skew-symmetric matrix given by

$$
\omega^{*}=\left[\begin{array}{ccc}
0 & -\omega_{z} & \omega_{y} \\
\omega_{z} & 0 & -\omega_{x} \\
-\omega_{y} & \omega_{x} & 0
\end{array}\right] .
$$

Furthermore, disturbance $F_{\mathrm{d}}(\omega(t), t)$ is assumed to be norm bounded:

$$
\left\|F_{d}(\omega(t), t)\right\| \leq k\|\omega\|,
$$

where $\|\cdot\|$ denotes the Euclidian vector norm and $k>0$ is a scalar. Using (1)-(3), the overall dynamics can be expressed as

$$
\begin{aligned}
& \dot{\theta}=f_{1}(\theta) \omega, \\
& \dot{\omega}=f_{2}(\omega)+g_{2} u+J^{-1} F_{\mathrm{d}}(\omega, t), \\
& y=\theta,
\end{aligned}
$$

where $f_{2}(\omega)=-J^{-1} \omega^{*} J \omega, g_{2}=J^{-1} D$, and

$$
f_{1}(\theta)=\left[\begin{array}{ccc}
0 & \sin \left(\theta_{\mathrm{r}}\right) & \cos \left(\theta_{\mathrm{r}}\right) \\
0 & \frac{\cos \left(\theta_{\mathrm{r}}\right)}{\cos \left(\theta_{\mathrm{p}}\right)} & -\frac{\sin \left(\theta_{\mathrm{r}}\right)}{\cos \left(\theta_{\mathrm{p}}\right)} \\
1-\tan \left(\theta_{\mathrm{p}}\right) \cos \left(\theta_{\mathrm{r}}\right) & \tan \left(\theta_{\mathrm{p}}\right) \sin \left(\theta_{\mathrm{r}}\right)
\end{array}\right] .
$$

\section{Attitude Tracking via Error Dynamics}

To formulate the attitude tracking problem, the error dynamics must be determined. To this end, we define error vector $e=\left[e_{\mathrm{p}} e_{\mathrm{y}} e_{\mathrm{r}}\right]^{\mathrm{T}}=\theta-\theta^{\mathrm{d}}$ such that

$$
\begin{gathered}
e_{\mathrm{p}}=\theta_{\mathrm{p}}-\theta_{\mathrm{p}}^{\mathrm{d}}, \\
e_{\mathrm{y}}=\theta_{\mathrm{y}}-\theta_{\mathrm{y}}^{\mathrm{d}}, \\
e_{\mathrm{r}}=\theta_{\mathrm{r}}-\theta_{\mathrm{r}}^{\mathrm{d}},
\end{gathered}
$$

where $\theta^{\mathrm{d}}$ is a bounded vector of the desired reference trajectories and assumed to have continuous first and second derivatives. Taking the time derivative of the error vector, the error dynamics can be written as

$$
\dot{e}=f(e) \omega-\dot{\theta}^{\mathrm{d}},
$$

where

$$
\begin{aligned}
& f(e) \\
& =\left[\begin{array}{ccc}
0 & \sin \left(e_{\mathrm{r}}+\theta_{\mathrm{r}}^{\mathrm{d}}\right) & \cos \left(e_{\mathrm{r}}+\theta_{\mathrm{r}}^{\mathrm{d}}\right) \\
0 & \frac{\cos \left(e_{\mathrm{r}}+\theta_{\mathrm{r}}^{\mathrm{d}}\right)}{\cos \left(e_{\mathrm{p}}+\theta_{\mathrm{p}}^{\mathrm{d}}\right)} & -\frac{\sin \left(e_{\mathrm{r}}+\theta_{\mathrm{r}}^{\mathrm{d}}\right)}{\cos \left(e_{\mathrm{p}}+\theta_{\mathrm{p}}^{\mathrm{d}}\right)} \\
1 & -\tan \left(e_{\mathrm{p}}+\theta_{\mathrm{p}}^{\mathrm{d}}\right) \cos \left(e_{\mathrm{r}}+\theta_{\mathrm{r}}^{\mathrm{d}}\right) & \tan \left(e_{\mathrm{p}}+\theta_{\mathrm{p}}^{\mathrm{d}}\right) \sin \left(e_{\mathrm{r}}+\theta_{\mathrm{r}}^{\mathrm{d}}\right)
\end{array}\right] .
\end{aligned}
$$

For state transformation, let us consider the dynamics $\left[\dot{e}^{\mathrm{T}} \dot{\omega}^{\mathrm{T}}\right]^{\mathrm{T}}$. To facilitate the design of the backstepping control law and enable the design of the high-gain observer, a change of variables is proposed to express the system in a feedback form. To this end, consider state vector $z(t)=\left[\begin{array}{ll}z_{1}^{\mathrm{T}} & z_{2}^{\mathrm{T}}\end{array}\right]^{\mathrm{T}}$ such that 


$$
\begin{aligned}
& z_{1}=e=\theta-\theta^{\mathrm{d}}, \\
& z_{2}=\dot{e}=f(e) \omega-\dot{\theta}^{\mathrm{d}}
\end{aligned}
$$

where subvector $z_{1}=e=\left[\begin{array}{lll}z_{1 \mathrm{p}} & z_{1 \mathrm{y}} & z_{1 \mathrm{r}}\end{array}\right]^{\mathrm{T}}$. Taking the time derivative of (10), the SCS dynamics in the new coordinates can be expressed as

$$
\begin{aligned}
& \dot{z}_{1}=z_{2}, \\
& \dot{z}_{2}=f_{z}+g_{z} u(t)+d(t), \\
& y_{z}=z_{1},
\end{aligned}
$$

where

$$
\begin{aligned}
f_{z}= & \dot{f}\left(z_{1}\right) f\left(z_{1}\right)^{-1} z_{2}+f\left(z_{1}\right) f_{2}(\omega) \\
& +\dot{f}\left(z_{1}\right) f\left(z_{1}\right)^{-1} \dot{\theta}^{\mathrm{d}}-\ddot{\theta}^{\mathrm{d}}, \\
g_{z}= & f\left(z_{1}\right) g_{2}, \\
d(t)= & f\left(z_{1}\right) J^{-1} F_{\mathrm{d}}(\omega, t),
\end{aligned}
$$

with

$$
\begin{aligned}
& \dot{f}\left(z_{1}\right)=\left[\begin{array}{ccc}
0 & 0 & 0 \\
0 & \frac{\sin \left(z_{1 \mathrm{p}}+\theta_{\mathrm{p}}^{\mathrm{d}}\right) \cos \left(z_{1 \mathrm{r}}+\theta_{\mathrm{r}}^{\mathrm{d}}\right)}{\cos ^{2}\left(z_{1 \mathrm{p}}+\theta_{\mathrm{p}}^{\mathrm{d}}\right)} & \frac{-\sin \left(z_{1 \mathrm{p}}+\theta_{\mathrm{p}}^{\mathrm{d}}\right) \sin \left(z_{1 \mathrm{r}}+\theta_{\mathrm{r}}^{\mathrm{d}}\right)}{\cos ^{2}\left(z_{1 \mathrm{p}}+\theta_{\mathrm{p}}^{\mathrm{d}}\right)} \\
0-\sec ^{2}\left(z_{1 \mathrm{p}}+\theta_{\mathrm{p}}^{\mathrm{d}}\right) \cos \left(z_{1 \mathrm{r}}+\theta_{\mathrm{r}}^{\mathrm{d}}\right) & \sec ^{2}\left(z_{1 \mathrm{p}}+\theta_{\mathrm{p}}^{\mathrm{d}}\right) \sin \left(z_{1 \mathrm{r}}+\theta_{\mathrm{r}}^{\mathrm{d}}\right)
\end{array}\right]\left(\dot{z}_{1 \mathrm{p}}+\dot{\theta}_{\mathrm{p}}^{\mathrm{d}}\right) \\
& +\left[\begin{array}{ccc}
0 & \cos \left(z_{1 \mathrm{r}}+\theta_{\mathrm{r}}^{\mathrm{d}}\right) & -\sin \left(z_{1 \mathrm{r}}+\theta_{\mathrm{r}}^{\mathrm{d}}\right) \\
0 & \frac{-\sin \left(z_{1 \mathrm{r}}+\theta_{\mathrm{r}}^{\mathrm{d}}\right)}{\cos \left(z_{1 \mathrm{p}}+\theta_{\mathrm{p}}^{\mathrm{d}}\right)} & \frac{-\cos \left(z_{1 \mathrm{r}}+\theta_{\mathrm{r}}^{\mathrm{d}}\right)}{\cos \left(z_{1 \mathrm{p}}+\theta_{\mathrm{p}}^{\mathrm{d}}\right)} \\
0 & \tan \left(z_{1 p}+\theta_{\mathrm{p}}^{\mathrm{d}}\right) \sin \left(z_{1 \mathrm{r}}+\theta_{\mathrm{r}}^{\mathrm{d}}\right) & \tan \left(z_{1 \mathrm{p}}+\theta_{\mathrm{p}}^{\mathrm{d}}\right) \cos \left(z_{1 \mathrm{r}}+\theta_{\mathrm{r}}^{\mathrm{d}}\right)
\end{array}\right]\left(\dot{z}_{1 \mathrm{r}}+\dot{\theta}_{\mathrm{r}}^{\mathrm{d}}\right) .
\end{aligned}
$$

Remark 2. The existence of $f\left(z_{1}\right)^{-1}$ is established by recognizing that the determinant of $f\left(z_{1}\right)$ is given by $\left|f\left(z_{1}\right)\right|=$ $-1 / \cos \left(z_{1 \mathrm{p}}+\theta_{\mathrm{p}}^{\mathrm{d}}\right)$. Given that angles are assumed to vary in a small range (Assumption 1$)$, the term $\cos \left(z_{1 \mathrm{p}}+\theta_{\mathrm{p}}^{\mathrm{d}}\right)$ is nonzero. Therefore, the determinant of $f\left(z_{1}\right)$ is bounded and $f\left(z_{1}\right)^{-1}$ exists.

Remark 3. The boundness of $f\left(z_{1}\right)$ is established by recognizing that the trigonometric functions $\sin (\cdot)$ and $\cos (\cdot)$ are bounded for all $\theta \in[0,2 \pi]$. Furthermore, as the angles are assumed to vary in a small range (Assumption 1), terms having the function $\cos (\cdot)$ are nonzero, and those having the function $\tan (\cdot)$ are bounded.

\section{Observer Design}

For many control design applications, some or even all the system states may not be measurable. Therefore, an observer can be used to estimate the states based on system measurements. In the proposed attitude control, a high-gain observer is adopted. Consider the dynamics in (11) in the form

$$
\begin{aligned}
& \dot{z}_{1}=z_{2}, \\
& \dot{z}_{2}=f(z, u, d), \\
& y_{o}=z_{1} .
\end{aligned}
$$

Assuming that the function $f(\cdot)$ is Lipschitz continuous and that the external disturbances vector $d(t)$ is bounded and differentiable, the corresponding high-gain observer is given by [18]

$$
\begin{aligned}
& \dot{\widehat{z}}_{1}=\widehat{z}_{2}+h_{1}\left(y_{o}-\hat{y}_{\mathrm{o}}\right), \\
& \dot{\vec{z}}_{2}=h_{2}\left(y_{o}-\widehat{y}_{\mathrm{o}}\right),
\end{aligned}
$$

where $h_{1}$ and $h_{2}$ are chosen such that matrix

$$
\left[\begin{array}{ll}
-h_{1} & 1 \\
-h_{2} & 0
\end{array}\right]
$$

obeys the Hurwitz criterion. Furthermore, the observer gains are chosen such that

$$
\begin{aligned}
& h_{1}=\frac{k_{1}}{\varepsilon}, \\
& h_{2}=\frac{k_{2}}{\varepsilon^{2}}
\end{aligned}
$$

where $k_{1}, k_{2}>0$ are scalars and $\varepsilon>0$ is a small number chosen to improve the convergence of the observer dynamics.

From the dynamics in (11), the term $f_{z}$ is locally Lipschitz continuous, and the reference trajectory $\theta^{\mathrm{d}}$ is bounded and assumed to be continuous in the first and second derivatives. Furthermore, the disturbance signal $d(t)$ defined in (12) is bounded. 


\section{Robust Backstepping Control Design}

In this section, a control design based on the backstepping technique is proposed for the SCS. Backstepping control is a systematic procedure based on the recursive application of Lyapunov functions [19]. The main result from this paper is presented below.

Proposition 4. The nonlinear dynamics of the uncertain SCS given by (5) are asymptotically stable under the following robust backstepping control law:

$$
u_{\mathrm{bs}}(t)=-\widehat{g}_{z}^{-1}\left(\widehat{f}_{z}+\alpha_{1} \widehat{z}_{2}+\alpha_{2} \zeta+R^{T} \zeta \eta(t)\right),
$$

where

$$
\eta(t)=\frac{k\left\|f\left(\widehat{z}_{1}\right)\right\|\left\|J^{-1}\right\|\|\widehat{\omega}\|}{\left\|\zeta^{T} R\right\|}+\alpha_{3},
$$

$\alpha_{1}, \alpha_{2}$, and $\alpha_{3}$ are positive scalars, $R \in \mathfrak{R}^{3 \times 3}$ is a symmetric positive definite matrix, $\zeta=z_{2}-\phi\left(z_{1}\right)$, with $\phi\left(z_{1}\right)=-\alpha_{1} z_{1}$, and the hat above some variables denotes estimated values.

Proof. Consider the nonlinear dynamical model given by (11). The design of the backstepping controller first considers $z_{2}$ as a virtual control variable for the dynamic equation of $\dot{z}_{1}(t)$. The objective is to design control law $\phi\left(z_{1}\right)=z_{2}$ such that $z_{1} \longrightarrow 0$ as $t \longrightarrow \infty$. Let $V_{1}=(1 / 2) z_{1}^{T} Q z_{1}$ be a Lyapunov function candidate, where $Q$ is a symmetric positive definite matrix. Taking the time derivative of $V_{1}$ along the dynamic trajectories of $\dot{z}_{1}(t)$ gives

$$
\dot{V}_{1}=z_{1}^{T} \mathrm{Q} z_{2} .
$$

Choosing $z_{2}=\phi\left(z_{1}\right)=-\alpha_{1} z_{1}$, where $\alpha_{1}>0$ is a design parameter, (20) becomes

$$
\dot{V}_{1}=-\alpha_{1} z_{1}^{T} Q z_{1}
$$

As $\dot{V}_{1}<0$, the dynamics of $\dot{z}_{1}$ are asymptotically stable. Next, the stability of the overall dynamics for (11) is determined. Define error vector $\zeta=z_{2}-\phi\left(z_{1}\right)=z_{2}+\alpha_{1} z_{1}$ and consider Lyapunov function candidate $V_{2}=V_{1}+(1 / 2) \zeta^{T} R \zeta$. Taking the time derivative of $V_{2}$ along the trajectories of (11) gives

$$
\begin{aligned}
\dot{V}_{2} & =\dot{V}_{1}+\zeta^{T} R \dot{\zeta} \\
& =-\alpha_{1} z_{1}^{T} Q z_{1}+\zeta^{T} R\left(f_{z}+\alpha_{1} z_{2}+g_{z} u(t)+d(t)\right)
\end{aligned}
$$

Substituting $d(t)$ and $u(t)$ for their equivalent forms in (12) and (18), respectively, yields

$$
\begin{aligned}
\dot{V}_{2} & =-\alpha_{1} z_{1}^{T} Q z_{1}+\zeta^{T} R\left(f_{z}+\alpha_{1} z_{2}\right. \\
& -g_{z} \widehat{g}_{z}^{-1}\left(\widehat{f}_{z}+\alpha_{1} \widehat{z}_{2}+\alpha_{2} \zeta+R^{T} \zeta \eta(t)\right) \\
& \left.+f\left(z_{1}\right) J^{-1} F_{\mathrm{d}}(\omega, t)\right)
\end{aligned}
$$

Motivated by the results in $[18,20]$ for the high-gain observer design, by choosing $\varepsilon$ small enough, the error between the system states and the estimated states converges exponentially to zero. Hence, the estimates $\widehat{g}_{z}, \widehat{f}_{z}, \widehat{z}_{2}$ converge to $g_{z}, f_{z}, z_{2}$ in a very short time. Therefore, we can assume that, for $t \geq T$, where $T$ is a very small time, expression (23) reduces to

$$
\begin{aligned}
\dot{V}_{2}= & -\alpha_{1} z_{1}^{T} Q z_{1}-\alpha_{2} \zeta^{T} R \zeta+\zeta^{T} R f\left(z_{1}\right) J^{-1} F_{\mathrm{d}}(\omega, t) \\
& -\zeta^{T} R R^{T} \zeta \eta(t) .
\end{aligned}
$$

Finally, from (4) bounding the exogenous signal $d(t)$, using (19) for $\eta(t)$, and considering that, for any symmetric positive definite matrix $P$, the quadratic form $x^{T} P x \geq \lambda_{\min }(P)\|x\|^{2}$, where $\lambda_{\min }(P)$ is the smallest eigenvalue of matrix $P,(24)$ is bounded as follows:

$$
\begin{aligned}
\dot{V}_{2} \leq & -\alpha_{1} \lambda_{\min }(Q)\left\|z_{1}\right\|^{2}-\alpha_{2} \lambda_{\min }(R)\|\zeta\|^{2} \\
& -\alpha_{3}\left\|\zeta^{T} R\right\|^{2} .
\end{aligned}
$$

As $\dot{V}_{2}<0$, the closed-loop system is asymptotically stable. Therefore, $z_{1}, \zeta \longrightarrow 0$ as $t \longrightarrow \infty$. Finally, from the transformation in (10), it can be easily shown that $\theta \longrightarrow \theta^{\mathrm{d}}$ as $t \longrightarrow \infty$. Thus, the proposed controller achieves asymptotic tracking.

\section{Simulation Results}

The nonlinear dynamics of the uncertain SCS in (5) were simulated under the application of the proposed robust backstepping control law given by (18) and (19). The external disturbances were set to

$$
\begin{aligned}
F_{\mathrm{d}} & (\omega, t) \\
& =0.5\left[\sin \left(\omega_{x}(t)\right) \sin \left(\omega_{y}(t)\right) \sin \left(\omega_{z}(t)\right)\right]^{\mathrm{T}},
\end{aligned}
$$

and the vector of reference trajectories to

$$
\begin{aligned}
& \theta^{\mathrm{d}}(t)=\left[\begin{array}{lll}
\theta_{\mathrm{p}}^{\mathrm{d}}(t) & \theta_{\mathrm{y}}^{\mathrm{d}}(t) & \theta_{\mathrm{r}}^{\mathrm{d}}(t)
\end{array}\right]^{\mathrm{T}} \\
& =-0.5[\sin (0.1 t) \sin (0.1 t) \sin (0.1 t)]^{T} \text {. }
\end{aligned}
$$

In addition, the initial conditions were set to

$$
\begin{aligned}
& {\left[\theta_{p}(0), \theta_{y}(0), \theta_{r}(0), \omega_{x}(0), \omega_{y}(0), \omega_{z}(0)\right]^{T}} \\
& \quad=[-0.8,0.4,0.7,0,0,0]^{T},
\end{aligned}
$$

the inertia matrix to

$$
J=\left[\begin{array}{ccc}
20 & 0.9 & 0 \\
0.9 & 17 & 0 \\
0 & 0 & 15
\end{array}\right]
$$

and the gains of the backstepping control to $\alpha_{1}=1.2, \alpha_{2}=$ 1.3 , and $\alpha_{3}=0.07$. The actuator distribution matrix $D$ and the positive definite matrix $R$ were chosen to be equal to identity $I_{3 \times 3}$. The simulations considered two cases: the case when all state variables are measurable and the case when the state variables are not measurable. A high-gain observer is used for the latter.

Figures 1-3 show the simulation results when all the state variables are measurable. Figure 1 shows the asymptotic convergence of the satellite angles towards the desired angle trajectories. As perfect tracking is achieved, the error trajectories between the actual and the desired angles tend to zero, as 

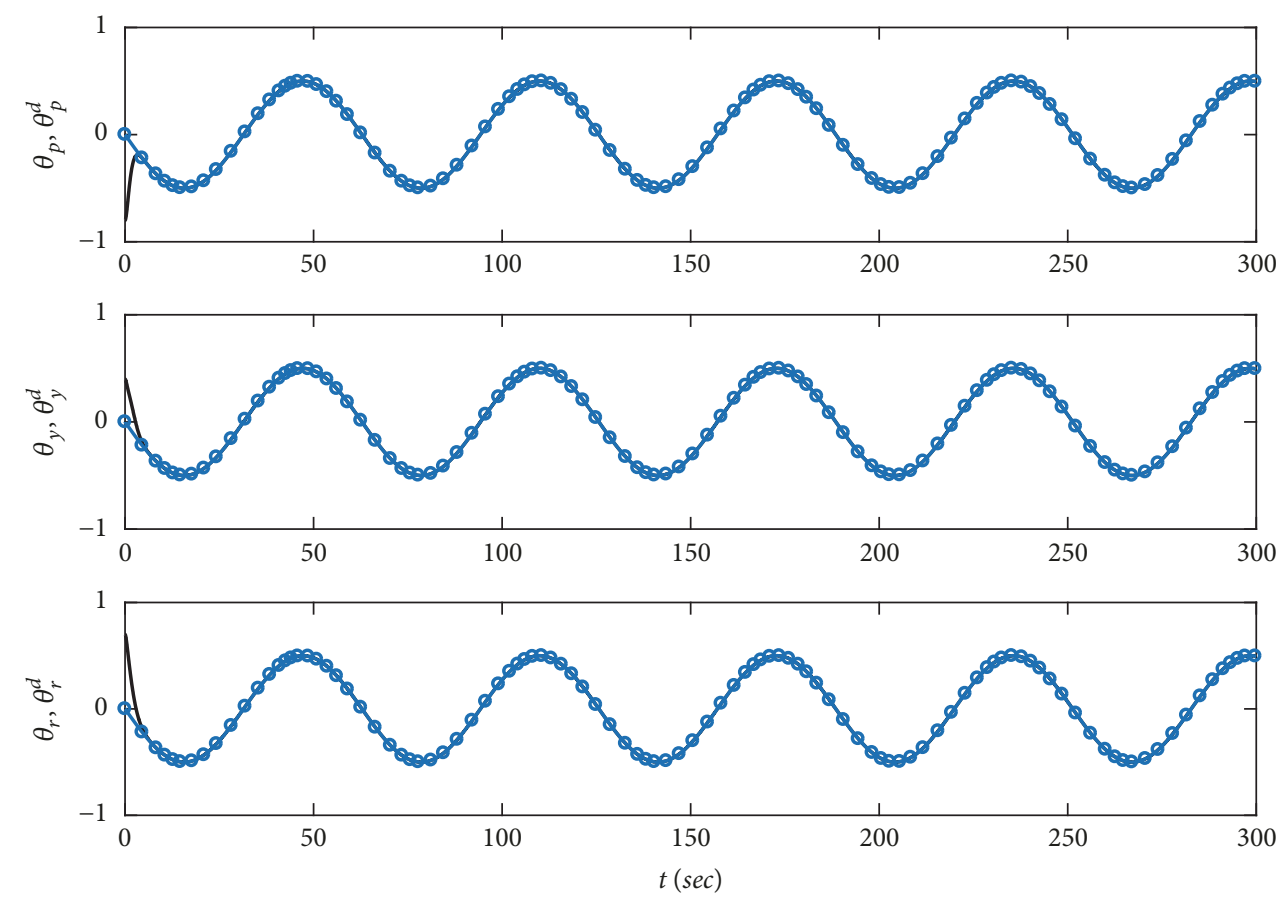

Figure 1: Pitch $\theta_{\mathrm{p}}$, yaw $\theta_{\mathrm{y}}$, and roll $\theta_{\mathrm{r}}$ angles of simulated SCS (solid lines) and desired trajectories (lines with circles) for the no observer case.
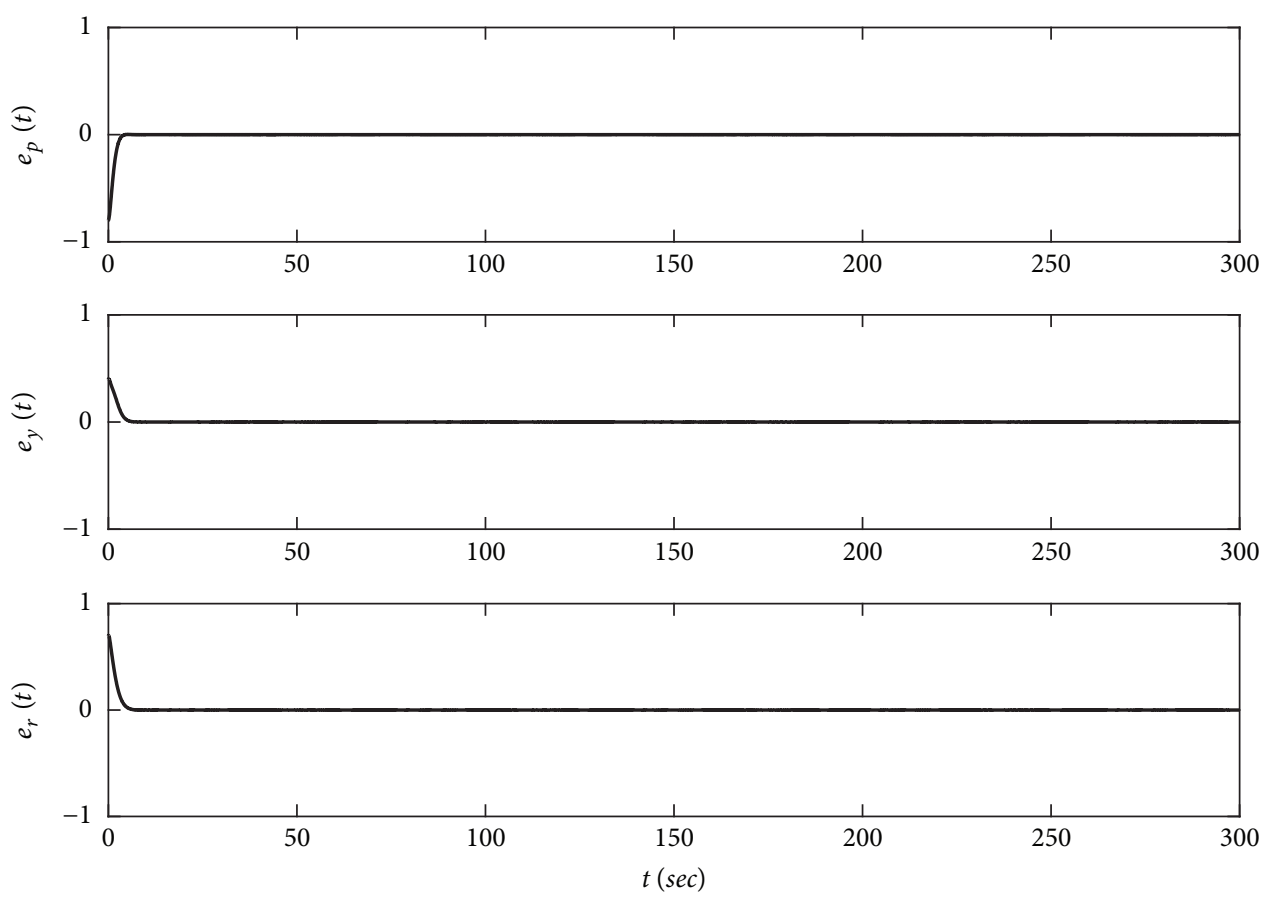

FIGURE 2: Error trajectories of pitch, yaw, and roll angles for the no observer case.

shown in Figure 2. Figure 3 depicts the control action exerted by the robust backstepping controller. These control signals describe the torques generated by the actuators causing the satellite to follow the desired path.

Next, the uncertain nonlinear dynamics of the SCS were simulated when a high-gain observer was required to estimate the system states. The initial conditions of the satellite system were set to

$$
\begin{aligned}
& {\left[\theta_{\mathrm{p}}(0), \theta_{\mathrm{y}}(0), \theta_{\mathrm{r}}(0), \omega_{x}(0), \omega_{y}(0), \omega_{z}(0)\right]^{\mathrm{T}}} \\
& =[-0.4,0.2,0.5,0,0,0]^{\mathrm{T}}
\end{aligned}
$$



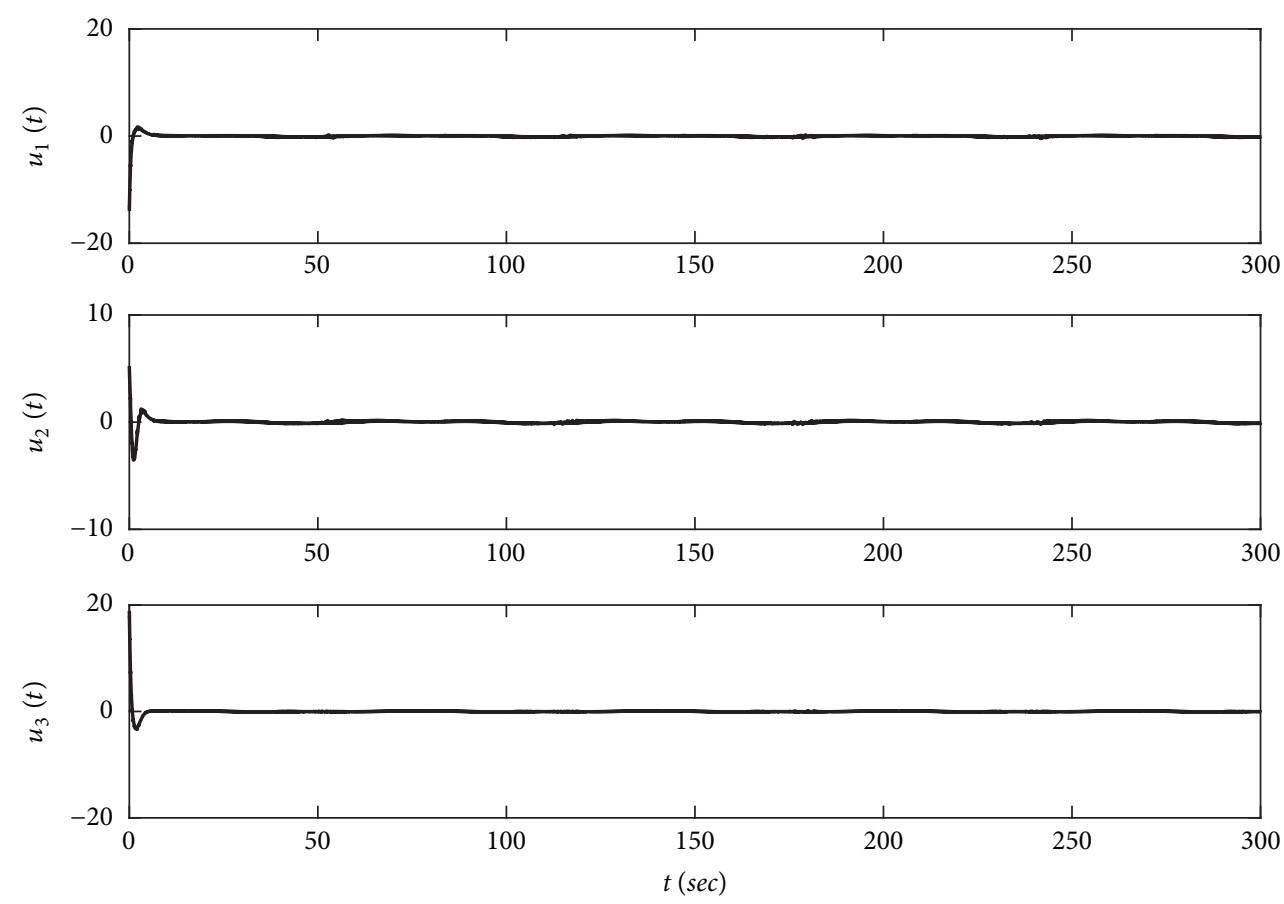

FIGURE 3: Time response of control inputs for the no observer case.
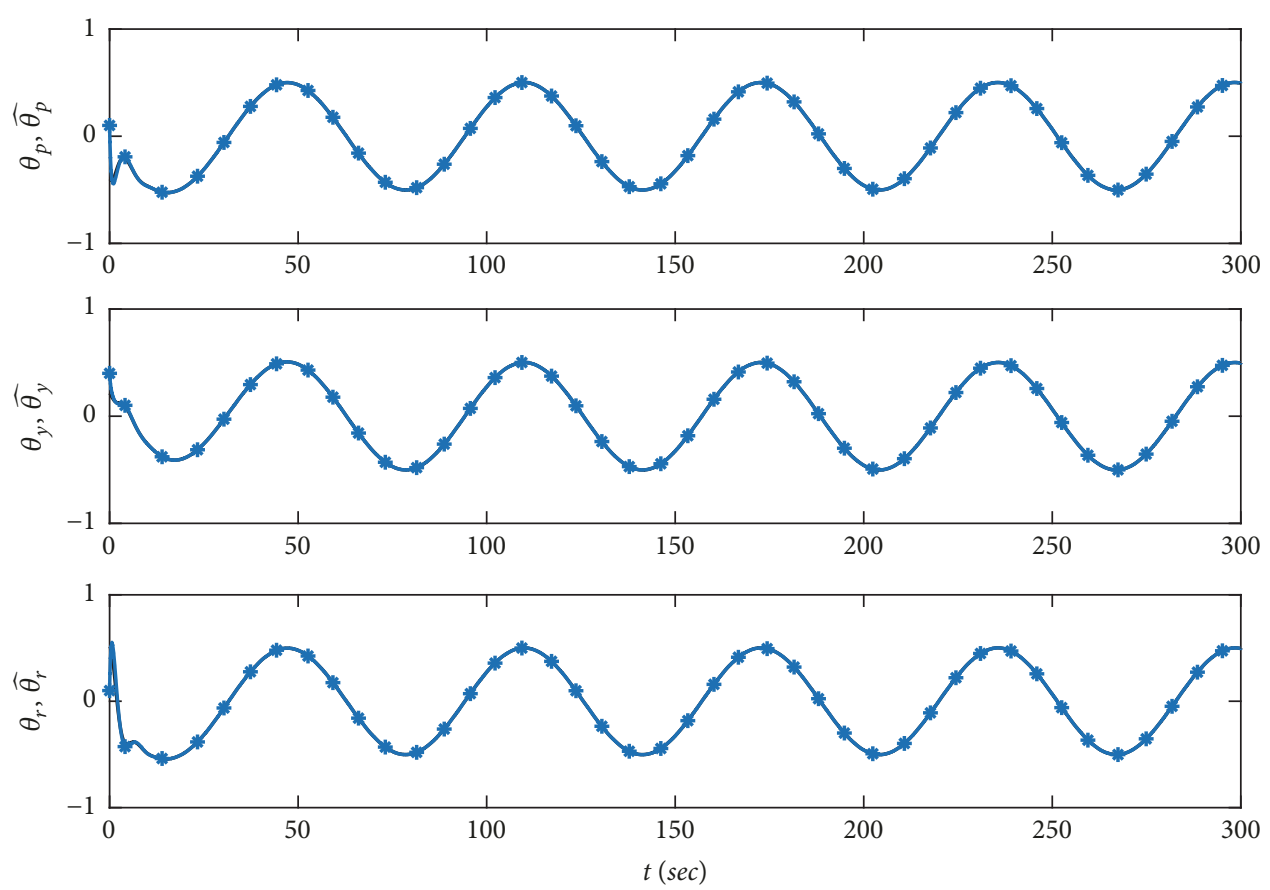

FIGURE 4: Pitch $\theta_{\mathrm{p}}$, yaw $\theta_{\mathrm{y}}$, and roll $\theta_{\mathrm{r}}$ of simulated SCS (solid lines) and observer estimated states (lines with asterisks).

and the observer initial conditions to

$$
\begin{aligned}
& {\left[\widehat{\theta}_{\mathrm{p}}(0), \widehat{\theta}_{\mathrm{y}}(0), \widehat{\theta}_{\mathrm{r}}(0), \widehat{\omega}_{x}(0), \widehat{\omega}_{y}(0), \widehat{\omega}_{z}(0)\right]^{\mathrm{T}}} \\
& \quad=[0.1,0.4,0.1,0.3,0.3,0.3]^{\mathrm{T}} .
\end{aligned}
$$

The robust backstepping controller gains were set to $\alpha_{1}=0.1$, $\alpha_{2}=0.5$, and $\alpha_{3}=0.04$, and the high-gain observer gains in (15) to $h_{1}=4$, and $h_{2}=4$.
Figures 4-8 show the simulation results with the highgain observer. Figures 4 and 5 show the effectiveness and fast convergence of the observer. Specifically, Figure 4 shows the asymptotic convergence of the estimated angles to the actual angles of the satellite system. Figure 5 depicts the asymptotic convergence of the estimated angular velocities to the actual angular velocities of the satellite system. The convergence of the satellite angles to the desired angle trajectories under 

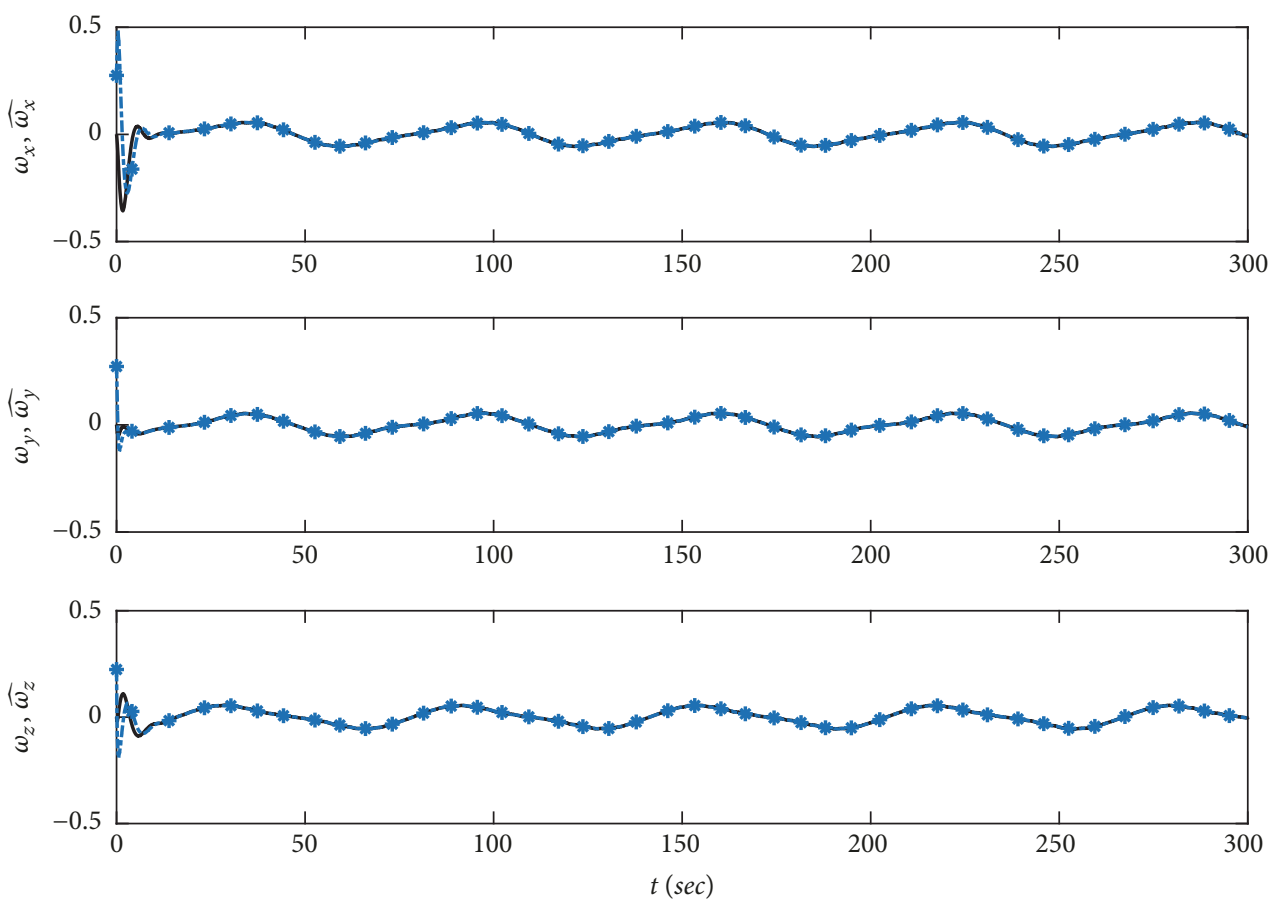

FIGURE 5: Angular velocities $\omega_{x}, \omega_{y}$, and $\omega_{z}$ of simulated SCS (solid lines) and observer estimated states (lines with asterisks).
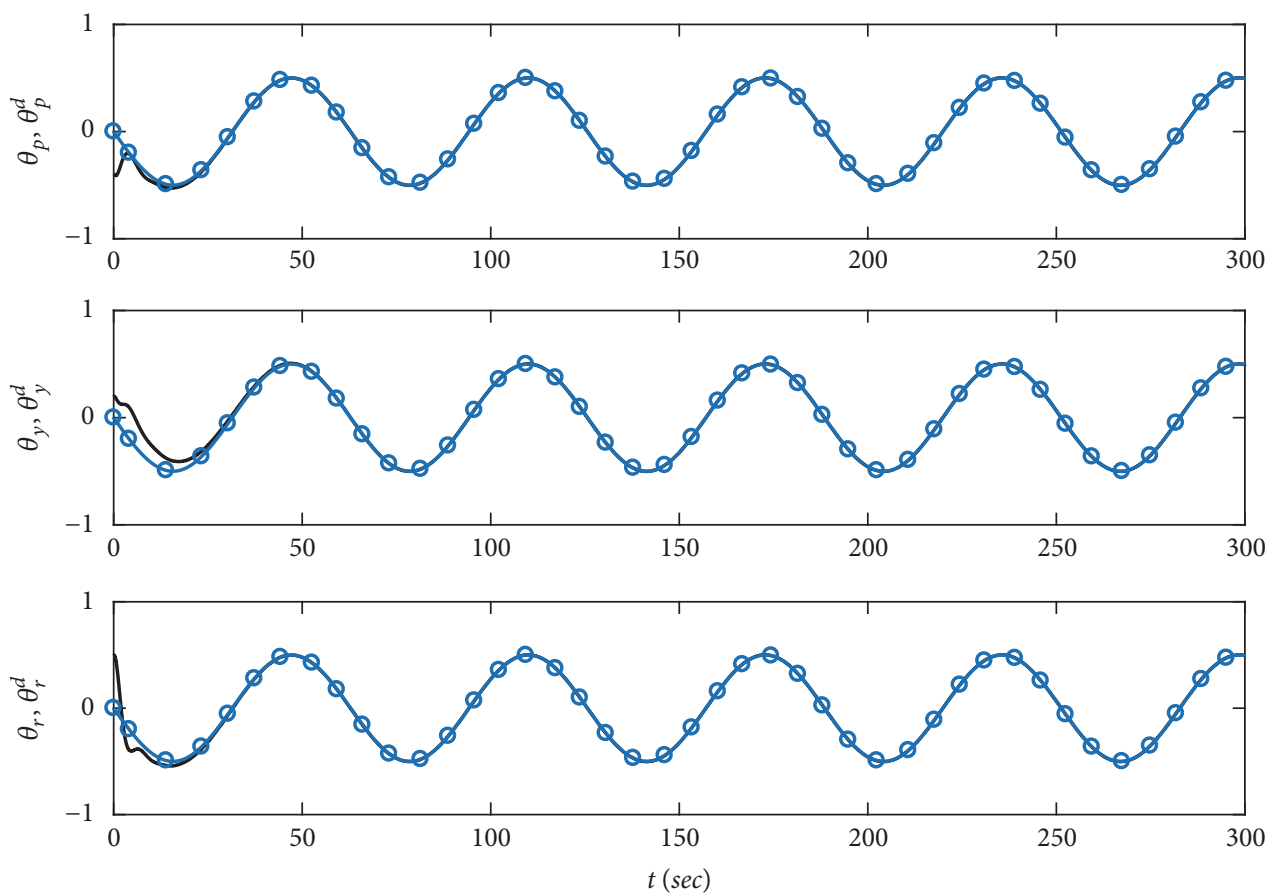

FIGURE 6: Pitch $\theta_{\mathrm{p}}$, yaw $\theta_{\mathrm{y}}$, and roll $\theta_{\mathrm{r}}$ of simulated SCS (solid lines) and desired reference trajectory (lines with circles) using the high-gain observer.

external disturbances is depicted in Figure 6. The error trajectories between actual and desired angles tend to zero, as shown in Figure 7. Finally, the evolution of the actuator control signals driving the satellite is depicted in Figure 8. The proposed robust backstepping control in (18) and (19) has successfully driven the satellite angles to their desired values, thus achieving asymptotic attitude tracking.

\section{Conclusion}

Attitude tracking for a class of SCS using a high-gain observer was addressed in this paper. The error dynamics of the SCS are first obtained, to then introduce a nonlinear transformation for expressing the system in feedback form, which enables the design of a high-gain observer. Then, 

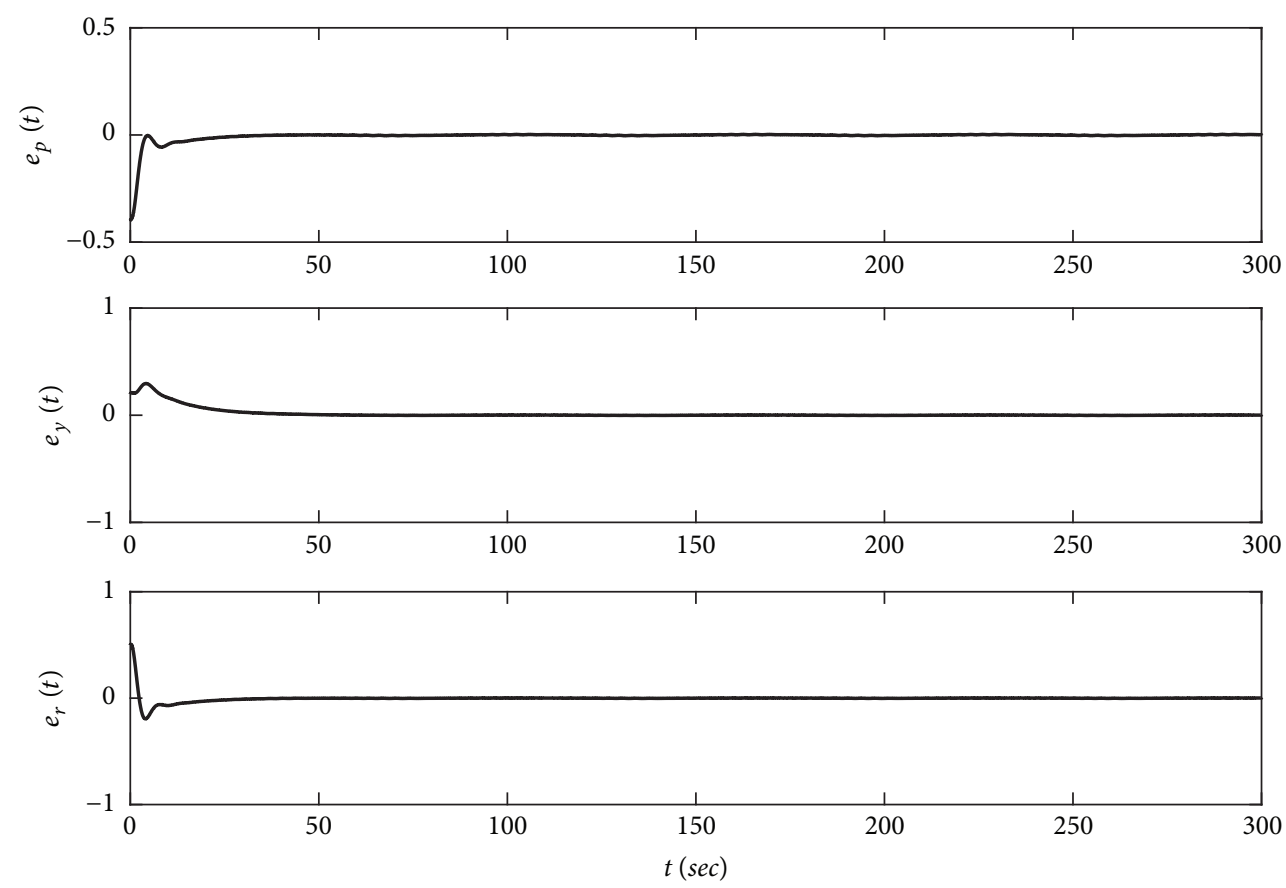

FIGURE 7: Error trajectories of pitch, yaw, and roll angles for the high-gain observer case.
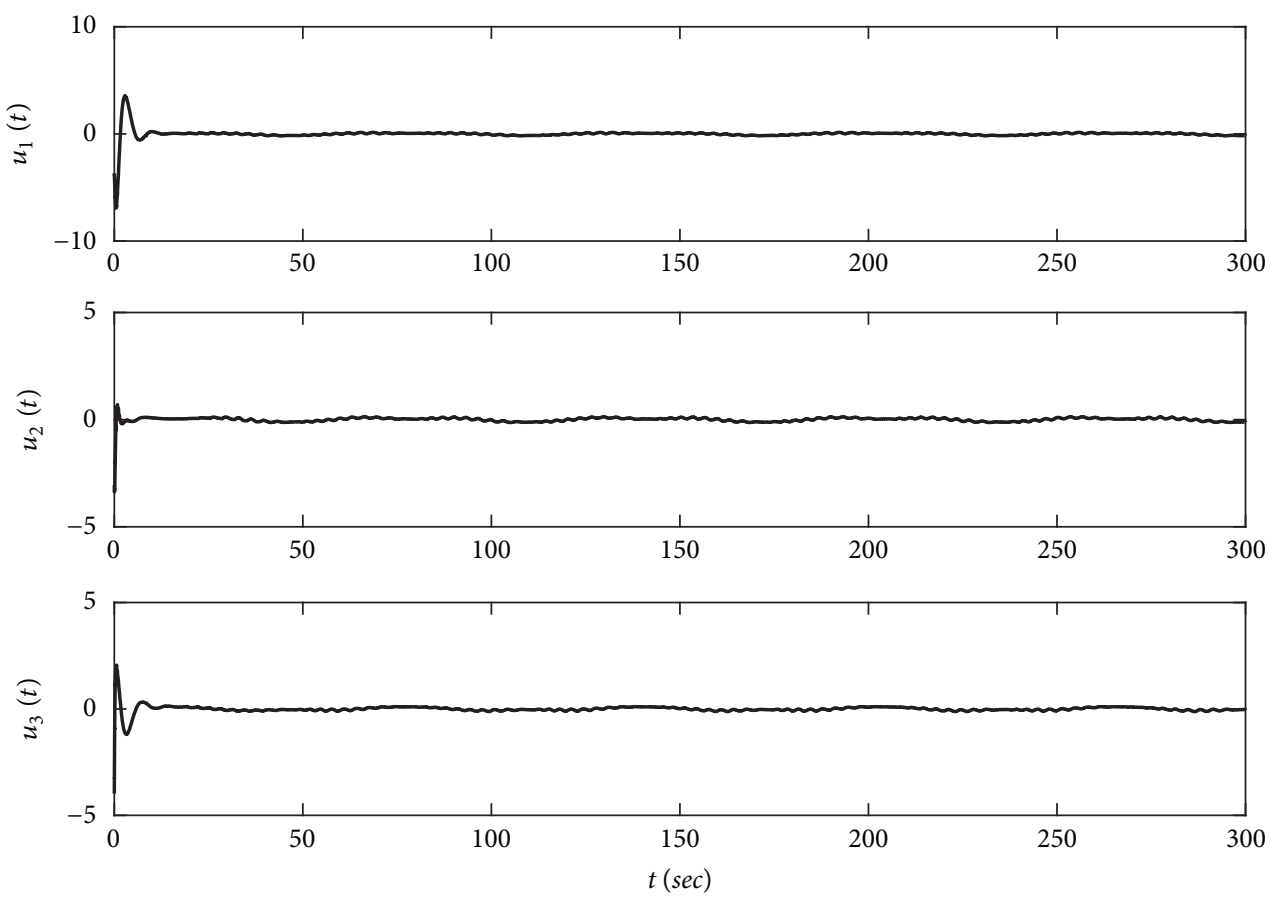

FIGURE 8: Time response of control inputs for the high-gain observer case.

a robust control based on backstepping control methodology was designed to drive the error dynamics to zero under exogenous perturbations, thus achieving attitude tracking. The asymptotic stability of the closed-loop system was established, and the simulation results showed the effectiveness of the proposed controller to achieve asymptotic convergence of the output trajectories to the desired reference values.

\section{Data Availability}

There are no research data taken from published work. Only references to other design techniques are included.

\section{Conflicts of Interest}

The authors declare that there are no conflicts of interest regarding the publication of this paper. 


\section{References}

[1] F. Menzione, R. Ferraro, A. Renga, and M. Grassi, "Multipurpose Earth Orbit Navigation System for autonomous orbit determination during satellite low thrust LEO-MEO transfer," in Proceedings of the 3rd IEEE International Workshop on Metrology for Aerospace (MetroAeroSpace), pp. 319-324, IEEE, Florence, Italy, 2016.

[2] J.-Z. Wang, Y.-Q. Hu, D.-Y. Yu, Z.-B. Cai, and Q.-X. Zhang, "Internal charging characteristics in typical navigation satellite orbits," IEEE Transactions on Plasma Sciences, vol. 46, no. 4, pp. 1010-1017, 2018.

[3] J. Jin, B. Park, Y. Park, and M.-J. Tahk, "Attitude control of a satellite with redundant thrusters," Aerospace Science and Technology, vol. 10, no. 7, pp. 644-651, 2006.

[4] B. A. Roberts, J. W. Kruk, T. B. Ake, T. S. Englar, B. F. Class, and D. M. Rovner, "Three-axis attitude control with two reaction wheels and magnetic torquer bars," AIAA Guidance, Navigation, and Control Conference and Exhibit, p. 5245, 2004.

[5] W. MacKunis, K. Dupree, S. Bhasin, and W. E. Dixon, "Adaptive neural network satellite attitude control in the presence of inertia and CMG actuator uncertainties," in Proceedings of the American Control Conference (ACC), pp. 2975-2980, IEEE, Seattle, Wash, USA, 2008.

[6] Y. Ma, B. Jiang, G. Tao, and Y. Cheng, "A direct adaptive actuator failure compensation scheme for satellite attitude control systems," Proceedings of the Institution of Mechanical Engineers, Part G: Journal of Aerospace Engineering, vol. 228, no. 4, pp. 542-556, 2014.

[7] S. Eshghi and R. Varatharajoo, "Nonsingular terminal sliding mode control technique for attitude tracking problem of a small satellite with combined energy and attitude control system (CEACS)," Aerospace Science and Technology, vol. 76, pp. 14-26, 2018.

[8] M. Wood and W.-H. Chen, "Attitude control of magnetically actuated satellites with an uneven inertia distribution," Aerospace Science and Technology, vol. 25, no. 1, pp. 29-39, 2013.

[9] D. K. Giri and M. Sinha, "Finite-time continuous sliding mode magneto-coulombic satellite attitude control," IEEE Transactions on Aerospace and Electronic Systems, vol. 52, no. 5, pp. 2397-2412, 2016.

[10] Y. Li, D. Ye, and Z. Sun, "Robust finite time control algorithm for satellite attitude control," Aerospace Science and Technology, vol. 68, pp. 46-57, 2017.

[11] X. Ma, F. Sun, H. Li, and B. He, "Attitude control of rigid body with inertia uncertainty and saturation input," Tsinghua Science and Technology, vol. 22, no. 1, pp. 83-91, 2017.

[12] M. TayyebTaher and S. M. Esmaeilzadeh, "Model predictive control of attitude maneuver of a geostationary flexible satellite based on genetic algorithm," Advances in Space Research, vol. 60, no. 1, pp. 57-64, 2017.

[13] M. Abdelrahman, I. Chang, and S.-Y. Park, "Magnetic torque attitude control of a satellite using the state-dependent Riccati equation technique," International Journal of Non-Linear Mechanics, vol. 46, no. 5, pp. 758-771, 2011.

[14] N. M. Horri, P. Palmer, and M. Roberts, "Gain-scheduled inverse optimal satellite attitude control," IEEE Transactions on Aerospace and Electronic Systems, vol. 48, no. 3, pp. 2437-2457, 2012.

[15] N. Sadati, N. D. Tehrani, and H. R. Bolandhemmat, "Multivariable adaptive satellite attitude controller design using RBF neural network," in Proceedings of IEEE International
Conference on Networking, Sensing and Control, vol. 2, pp. 11891194, IEEE, 2004.

[16] H. Lee and Y. Kim, "Fault-tolerant control scheme for satellite attitude control system," IET Control Theory and Applications, vol. 4, no. 8, pp. 1436-1450, 2010.

[17] B. Xiao, Q.-L. Hu, and G. Ma, "Adaptive sliding mode backstepping control for attitude tracking of flexible spacecraft under input saturation and singularity," Proceedings of the Institution of Mechanical Engineers, Part G: Journal of Aerospace Engineering, vol. 224, no. 2, pp. 199-214, 2010.

[18] H. Khalil and L. Praly, "High-gain observers in nonlinear feedback control," International Journal of Robust and Nonlinear Control, vol. 24, no. 6, pp. 993-1015, 2014.

[19] H. J. Marquez, Nonlinear Control Systems: Analysis And Design, vol. 1, Wiley Interscience, Hoboken, NJ, USA, 2003.

[20] H. K. Khalil, "High-gain observers in feedback control: application to permanent magnet synchronous motors," IEEE Control Systems Magazine, vol. 37, no. 3, pp. 25-41, 2017. 


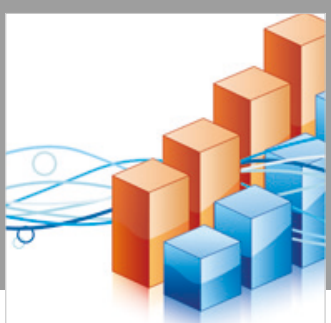

Advances in

Operations Research

\section{-n-m}
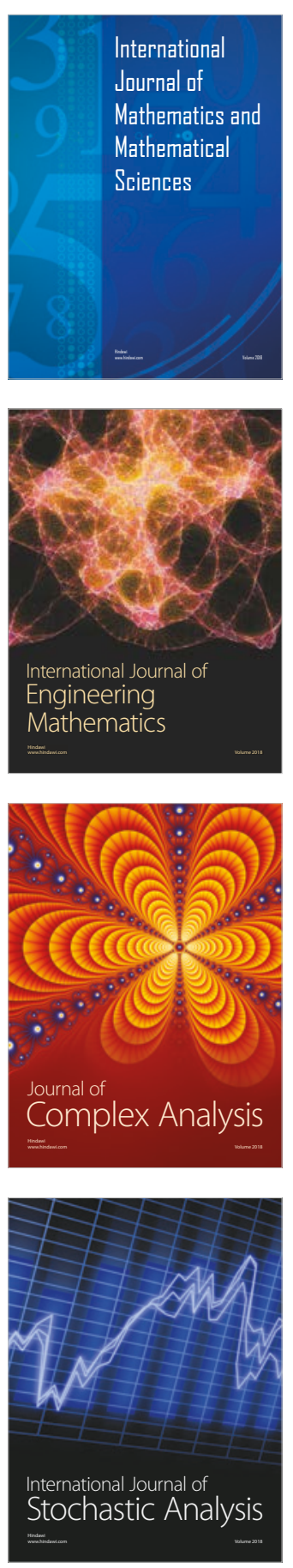
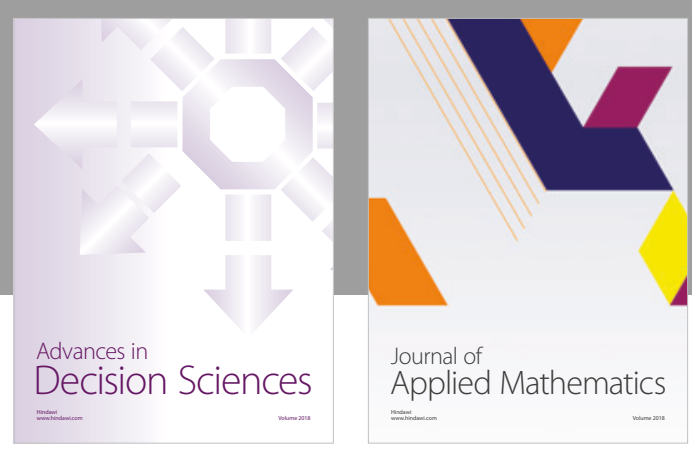

Journal of

Applied Mathematics
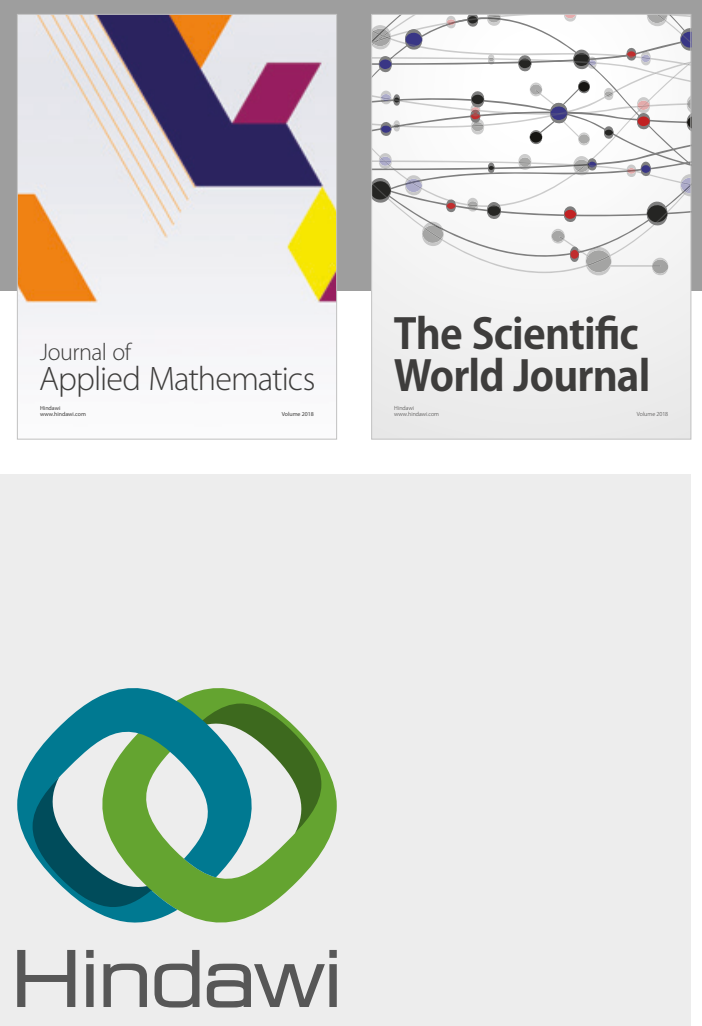

Submit your manuscripts at

www.hindawi.com

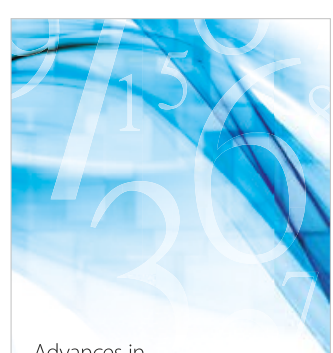

Advances in
Numerical Analysis
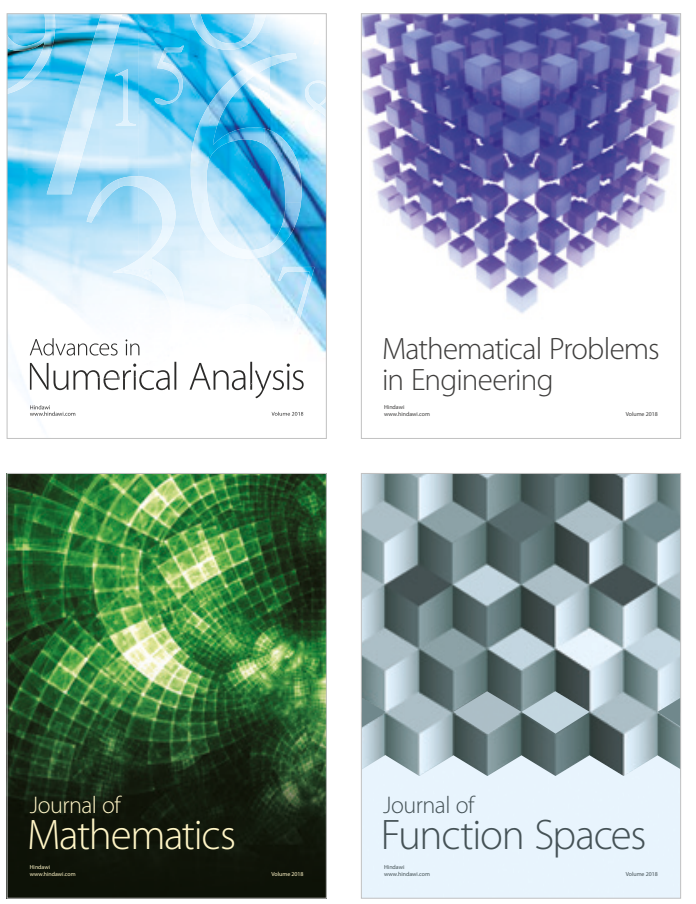

Mathematical Problems in Engineering

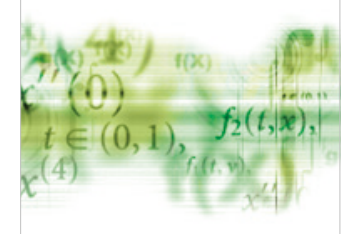

International Journal of

Differential Equations

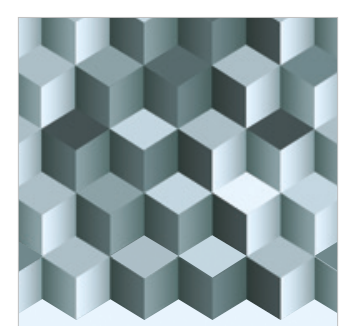

Journal of

Function Spaces

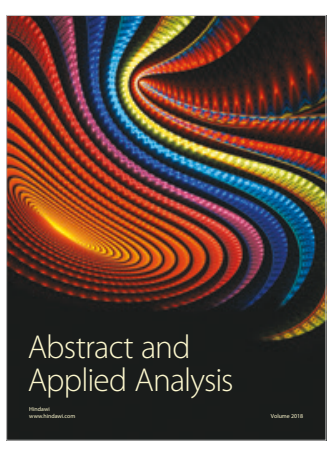

The Scientific

World Journal

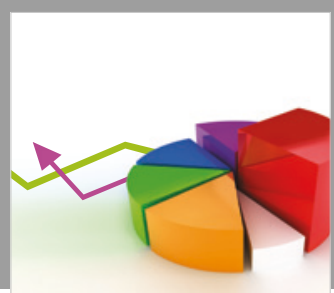

Journal of

Probability and Statistics
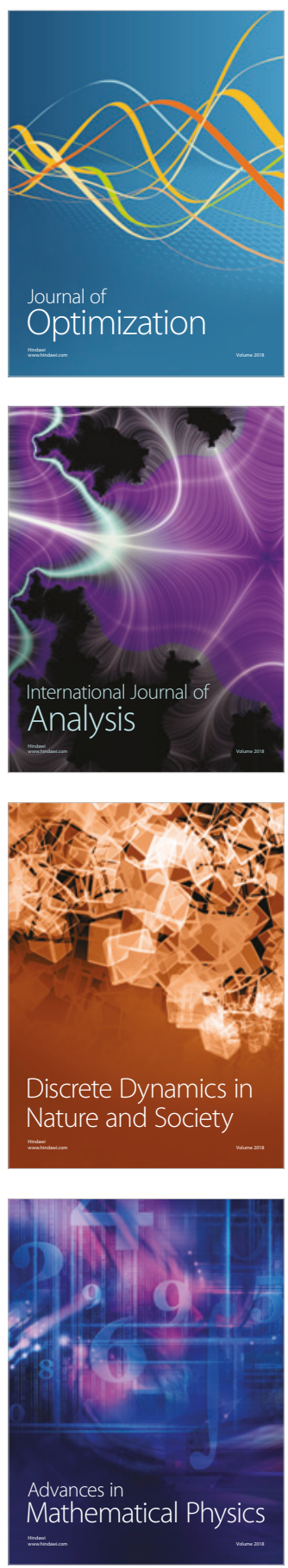